Research Article

\title{
A Modal-Based Kalman Filter Approach and OSP Method for Structural Response Reconstruction
}

\author{
Zhenrui Peng $(\mathbb{D}$, Kangli Dong $(\mathbb{D}$, and Hong Yin \\ School of Mechatronic Engineering, Lanzhou Jiaotong University, Lanzhou 730070, China \\ Correspondence should be addressed to Zhenrui Peng; pengzr@mail.lzjtu.cn
}

Received 28 February 2019; Revised 7 May 2019; Accepted 22 May 2019; Published 16 June 2019

Academic Editor: Andrea Spaggiari

Copyright (C) 2019 Zhenrui Peng et al. This is an open access article distributed under the Creative Commons Attribution License, which permits unrestricted use, distribution, and reproduction in any medium, provided the original work is properly cited.

\begin{abstract}
The objective of the work is experimental validation and optimal experimental design for structural response reconstruction. A modal-based Kalman filter approach based on excitation identification Kalman filter is proposed for response reconstruction and excitation estimation of structures by using noisy acceleration and strain measurements. Firstly, different filters are introduced and discussed. Secondly, to avoid single type sensors, a displacement reconstruction based on modal method is introduced into the proposed approach. Thirdly, the backward sequential algorithm is given to obtain the optimal sensor locations. It is shown that the proposed method can avoid the divergency in the estimated process of excitation and displacements as a result of incomplete measurements. Reasonable estimates of strains, displacements, velocities, accelerations, and excitations of structures can be accomplished with few acceleration sensors and strain gauges.
\end{abstract}

\section{Introduction}

This paper contributes to the problem of response reconstruction and excitation estimation of structures, which is given serious attention these years. Considering that it is difficult or sometimes impossible to monitor displacement and velocity information of structures, the unknown response reconstruction method is necessary to be studied. It is important to estimate the time history of different kinds of inputs in practice. The experimental validation and optimal experimental design for structural response reconstruction are considered as the problems to solve in this work.

The first type of response reconstruction approaches can be classified as transmissibility approach [1], which has been extended to $\mathrm{N}$ degrees of freedom (DOFs) systems by Ewins and Liu [2]. It is initially defined as the ratio of the response amplitude to the harmonic excitation amplitude for a single DOF system. Law et al. [3] performed dynamic response reconstruction and extended it to the wavelet domain [4] based on the generalized transmissibility concept. The implementation of transmissibility concept requires the known excitations, and it is only efficient for single type of response.

The second is via empirical mode decomposition (EMD) approach [5]. Using EMD approach, the measured responses are decoupled into intrinsic mode functions (IMFs), which are applied to estimate the response at unmeasured locations. Wan et al. [6] combined transmissibility with EMD approach to make it applicable for structures which have closely spaced modes of vibration. However, this method relies on signal decomposition ability of the algorithms heavily to achieve high-quality reconstructed response, because EMD approach may not achieve a great decomposition effect for different kinds of responses.

The third is based on different filters, which have been applied to engineering field in recent years [7-9]. There are well-proposed algorithms to solve both linear [10] and nonlinear [11] problems. A recursive three-step filter was proposed by Gillijns and De Moor [12] for unbiased minimum-variance state estimation and it used least square method to implement the estimation of the inputs, which has been used by Zhang and $\mathrm{Xu}$ [13] to reconstruct 
unmeasured responses and excitations of structures. Julier and Uhlmann [14] proposed the unscented Kalman filter (UKF), and Eftekhar Azam et al. [15] proposed a parallel implementation of the UKF. Ching et al. [16] compared the performance of the EKF with that of the particle filter (PF) applied to a linear multistorey shear building. Chatzi and Smyth [17], Eftekhar Azam et al. [18] compared the performance of the UKF and PF applied to nonlinear constitutive models. Nasr and Saad [19] proposed a GA-Ensemble Kalman filter (G-EKF).

Single input estimation methods based on structure response have also been proposed recently. Lai et al. present a parametric study on the sequential deconvolution input reconstruction (SDR) method [20], an explicit fourth-order Runge-Kutta method [21], and wavelet Galerkin method [22], respectively. The reconstruction of inputs from measured outputs is examined [23]. Aucejo and De Smet [24] fully exploited the Bayesian framework to provide, from a Markov chain Monte Carlo algorithm, credible intervals and other statistical measures for all the parameters of the force reconstruction problem. A monotonic two-step iterative shrinkage/thresholding (MTWIST) algorithm was proposed [25] to find the sparse solution to such an under determined model from highly incomplete and inaccurate measurements. A hierarchical Bayesian method was proposed [26], which does not require prior knowledge on the force nature. A power series polynomial model was utilized to model the nonlinear restoring force of a chain-like nonlinear multidegree-of-freedom structure [27]. $\mathrm{Xu}$ and $\mathrm{Ou}$ [28] proposed an identification method of multisource dynamic loads based on independent component analysis.

Most of the above methods require full-state measurements or different kinds of sensors to achieve accurate responses and excitations of the structure. However, in practice the displacements and velocities are difficult, or even sometimes impossible to be measured, which is the common case in structure dynamics. It is promising to use single-kind measurement to obtain multiple response signals. Liu et al. [29] used an adapted model to remedy the issue in which Kalman filter requires full-state measurements. Hwang et al. [30] proposed a Kalman-based method, in which the estimation of the states and excitations is coupled, and it does not require full measurements. Lourens et al. [31] proposed an augmented Kalman filter-based estimator, in which the states are augmented with the unknown excitations in order to estimate them at the same time. By using acceleration measurements, a Kalman filter with dummy measurements was proposed by Naets et al. [32] to avoid the so-called drift during the estimation process, which is similar to the one proposed by Chatzi and Fuggini [33] for stabilizing tracking for civil structure monitoring purposes. A dual Kalman filter approach was proposed by Eftekhar Azam et al. [34] to reconstruct the response of the Pirelli Tower under large inputs using acceleration measurements only, and the experimental validation of the proposed method was implemented [35]. All these methods are based on the improved filters to avoid using the uncommon measurement; however, the connections among different types of responses are ignored.
In order to obtain an easy-to-implement methodology, a displacement estimation based on modal method [36] was introduced into the proposed approach to avoid the drift effect caused by incomplete measurements. By experimental validation and optimal experimental design, the optimal sensor placement scheme was obtained. This method considers the connection between displacement and strain responses of the structure and uses a two-step approach to implement the reconstruction of responses, which would be a practical structural health monitoring method for high size civil structures.

The outline of the paper is as follows. Section 2 introduces the theories of the response reconstruction problem, Kalman filter, and modal method. Section 3 gives the frame of the proposed method. In Section 4, different types of inputs are applied to a two-dimensional truss structure to prove the validity of the proposed method. Section 5 provides an experimental example of cantilever beam to show efficiency of the proposed method.

\section{Background}

2.1. Mathematical Formulation of the Problem. A linear structural dynamics modeled by $n$ DOFs is typically formulated using the following continuous-time second-order differential equation:

$$
\mathbf{M} \ddot{\mathbf{z}}(t)+\mathbf{C} \dot{\mathbf{z}}(t)+\mathbf{K z}(t)=\mathbf{L} \mathbf{u}(t),
$$

where $\mathbf{M} \in \mathbb{R}^{n \times n}, \mathbf{C} \in \mathbb{R}^{n \times n}$, and $\mathbf{K} \in \mathbb{R}^{n \times n}$ stand for the mass, damping, and stiffness matrix, respectively; $\mathbf{z}(t) \in \mathbb{R}^{n}$ denotes the nodal displacement vector; $\mathbf{L} \in \mathbb{R}^{n \times p}$ is the influence matrix; $\mathbf{u}(t) \in \mathbb{R}^{p}$ denotes the inputs vector, which contains $n$ inputs.

Consider the undamped eigenvalue analysis corresponding to equation (1)

$$
\mathrm{K} \boldsymbol{\Phi}=\mathbf{M} \boldsymbol{\Phi} \omega_{0}^{2} .
$$

Transform the system of equation (1) using the following mapping:

$$
\mathbf{z}(t)=\boldsymbol{\Phi} \mathbf{q}(t),
$$

where $\mathbf{q}(t) \in \mathbb{R}^{p}$ is the vector of modal coordinates and $\Phi \in \mathbb{R}^{n \times p}$ is the displacement mode shape matrix. If massnormalized eigenvectors are used and the proportional damping is assumed, considering $\boldsymbol{\Phi}^{T} \mathbf{M} \boldsymbol{\Phi}=\mathbf{I}, \boldsymbol{\Phi}^{T} \mathbf{K} \boldsymbol{\Phi}=\omega_{0}^{2}$, and $\boldsymbol{\Phi}^{T} \mathbf{C} \boldsymbol{\Phi}=2 \xi \omega_{0}$, equation (1) can be further rewritten as:

$$
\ddot{\mathbf{q}}+2 \xi \omega_{0} \dot{\mathbf{q}}+\omega_{0}^{2} \mathbf{q}=\boldsymbol{\Phi}^{T} \mathbf{L u},
$$

where $\omega_{0} \in \mathbb{R}^{n \times n}$ and $\xi \in \mathbb{R}^{n \times n}$ denote the diagonal matrices containing the eigen frequency $\omega_{i}$ and the modal damping ratio $\xi_{i}$, respectively.

Equation (1) can be transformed into a discrete-time state space form [37]:

$$
\begin{aligned}
\mathbf{z}_{k+1} & =\mathbf{A} \mathbf{z}_{k}+\mathbf{B} \mathbf{u}_{k}, \\
\mathbf{y}_{k} & =\mathbf{C} \mathbf{z}_{k}+\mathbf{D} \mathbf{u}_{k},
\end{aligned}
$$

where $\mathbf{y}_{k}$ is the observation vector, and 


$$
\begin{aligned}
& \mathbf{A}=e^{\left[\begin{array}{cc}
\mathbf{0} & \mathbf{I} \\
-\omega_{0}^{2} & -2 \xi \omega_{0}
\end{array}\right] \Delta t,} \\
& \mathbf{B}=\left[\begin{array}{cc}
\mathbf{0} & \mathbf{I} \\
-\omega_{0}^{2} & -2 \xi \omega_{0}
\end{array}\right]^{-1}(\mathbf{A}-\mathbf{I})\left[\begin{array}{c}
\mathbf{0} \\
\boldsymbol{\Phi}^{T} \mathbf{L}
\end{array}\right], \\
& \mathbf{C}=\left[\begin{array}{cc}
\boldsymbol{\Phi} & \mathbf{0} \\
\mathbf{0} & \boldsymbol{\Phi} \\
-\boldsymbol{\Phi} \omega_{0}^{2} & -2 \boldsymbol{\Phi} \xi \omega_{0}
\end{array}\right], \\
& \mathbf{D}=\left[\begin{array}{c}
0 \\
0 \\
-\boldsymbol{\Phi} \boldsymbol{\Phi}^{T} \mathbf{L}
\end{array}\right],
\end{aligned}
$$

where $\mathbf{A} \in \mathbb{R}^{2 n \times 2 n}, \mathbf{B} \in \mathbb{R}^{2 n \times 2 n}, \mathbf{C} \in \mathbb{R}^{m \times 2 n}$, and $\mathbf{D} \in \mathbb{R}^{m \times p}$ represent the state matrix, input matrix, output matrix, and transmission matrix, respectively; I denotes the identity matrix with appropriate dimension; and $\Delta t$ is the sampling interval.

Considering the modeling errors and the measurement errors, equation (5) can be rewritten as

$$
\begin{aligned}
\mathbf{z}_{k+1} & =\mathbf{A} \mathbf{z}_{k}+\mathbf{B} \mathbf{u}_{k}+\mathbf{w}_{k}, \\
\mathbf{y}_{k}^{m} & =\mathbf{C}^{m} \mathbf{z}_{k}+\mathbf{D}^{m} \mathbf{u}_{k}+\mathbf{v}_{k},
\end{aligned}
$$

where $\mathbf{y}_{k}^{m}$ is the sensor measurements; $\mathbf{C}^{m}$ and $\mathbf{D}^{m}$ are submatrices of $\mathbf{C}$ and $\mathbf{D}$, respectively; $m$ denotes the rows which will be measured; $\mathbf{w}_{k} \in \mathbb{R}^{2 n} \sim N\left(0, \mathbf{Q}_{\mathrm{z}}\right)$ and $\mathbf{v}_{k} \in \mathbb{R}^{m} \sim N(0, \mathbf{R})$ denote the independent, white, and normally distributed modeling error and measurement error, respectively; and $\mathbf{Q}_{z}$ and $\mathbf{R}$ are the variance matrices of modeling error and measurement error, respectively.

\subsection{Response Reconstruction and Excitation Estimation with} Kalman Filter. Response reconstruction and unknownexcitation estimation have attracted large attention in recent years. A three-step filter (excitation identification Kalman filter, EIKF) containing both least square method and Kalman filter was proposed by Gillijns and De Moor [12] for unbiased minimum-variance state and excitation estimation, which was widely used for solving the above problem [13]. A new method (robust two-stage Kalman filter, RTSKF) was developed by Hsieh [38] for the state estimation of linear time-varying discrete systems with unknown inputs. The general schemes of the two different filters are detailed in Algorithm 1 and Algorithm 2, respectively.

Here, the two filters aim to solve the problem of estimating the state of a linear time-invariant discrete system in the presence of unknown inputs. EIKF contains three basic steps: the input prediction stage, the measurement updating stage, and the time updating stage. The Kalman gain is then the optimal weighting function which gives the best balance between the time updating and the measurement updating. The two steps behind are based on the Kalman filter, while the principle of least square method is used in the prediction of input. The reconstructed responses $\widehat{\mathbf{y}}_{k}$ can be calculated
(1) Initialization at time $t_{0}$ :

(2) $\widehat{\mathbf{z}}_{0}=\mathbb{E}\left[\mathbf{z}_{0}\right]$

(3) $\mathbf{P}_{0}=\mathbb{E}\left[\left(\mathbf{z}_{0}-\widehat{\mathbf{z}}_{0}\right)\left(\mathbf{z}_{0}-\widehat{\mathbf{z}}_{0}\right)^{T}\right]$

(4) while $k=1, \ldots, N_{t}$ do

(5) Input prediction stage:

(6) $\quad \widetilde{\mathbf{R}}_{k}=\mathbf{C}^{m} \mathbf{P}_{k}^{z-} \mathbf{C}^{m T}+\mathbf{R}$

(7) $\quad \mathbf{M}_{k}=\left(\mathbf{D}^{m T} \widetilde{\mathbf{R}}_{k}^{-1} \mathbf{D}^{m}\right)^{-1} \mathbf{D}^{m T} \widetilde{\mathbf{R}}_{k}^{-1}$

(8) $\quad \widehat{\mathbf{u}}_{k}=\mathbf{M}_{k}\left(\mathbf{y}_{k}-\mathbf{C}^{m} \mathbf{z}_{k}^{-}\right)$

(9) $\quad \mathbf{P}_{k}^{u}=\left(\mathbf{D}^{m T} \widetilde{\mathbf{R}}_{k}^{-1} \mathbf{D}^{m}\right)^{-1}$

(10) Measurement updating stage:

(11) $\quad \mathbf{K}_{k}=\mathbf{P}_{k}^{z-} \mathbf{C}^{m T} \widetilde{\mathbf{R}}_{k}^{-1}$

(12) $\widehat{\mathbf{z}}_{k}^{+}=\mathbf{z}_{k}^{-}+\mathbf{K}_{k}\left(\mathbf{y}_{k}-\mathbf{C}^{m} \mathbf{z}_{k}^{-}-\mathbf{D}^{m} \widehat{\mathbf{u}}_{k}\right)$

(13) $\quad \mathbf{P}_{k}^{z+}=\mathbf{P}_{k}^{z-}-\mathbf{K}_{k}\left(\widetilde{\mathbf{R}}_{k}-\mathbf{D}^{m} \mathbf{P}_{k}^{u} \mathbf{D}^{m T}\right) \mathbf{K}_{k}^{T}$

(14) $\quad \mathbf{P}_{k}^{z u}=\left(\mathbf{P}_{k}^{u z}\right)^{T}=-\mathbf{K}_{k} \mathbf{D}^{m} \mathbf{P}_{k}^{u}$

(15) Time updating stage:

(16) $\quad \mathbf{z}_{k+1}^{-}=\mathbf{A} \widehat{\mathbf{z}}_{k}^{+}+\mathbf{B} \widehat{\mathbf{u}}_{k}$

(17) $\quad \mathbf{P}_{k+1}^{z-}=\left[\begin{array}{ll}\mathbf{A} & \mathbf{B}\end{array}\right]\left[\begin{array}{cc}\mathbf{P}_{k}^{z+} & \mathbf{P}_{k}^{z u} \\ \mathbf{P}_{k}^{u z} & \mathbf{P}_{k}^{u}\end{array}\right]\left[\begin{array}{l}\mathbf{A}^{T} \\ \mathbf{B}^{T}\end{array}\right]+\mathbf{Q}_{z}$

(18) $\quad \widehat{\mathbf{y}}_{k}=\mathbf{C} \widehat{\mathbf{z}}_{k}^{+}+\mathbf{D} \widehat{\mathbf{u}}_{k}$

(19) end while

(20) Obtain $\widehat{\mathbf{y}}_{k}$, $\widehat{\mathbf{z}}_{k}^{+}$and $\widehat{\mathbf{u}}_{k}$.

Algorithm 1: EIKF.

$$
\begin{aligned}
& \text { (1) Initialization at time } t_{0} \text { : } \\
& \text { (2) } \widehat{\mathbf{z}}_{0}=\mathbb{E}\left[\mathbf{z}_{0}\right] \\
& \text { (3) } \mathbf{P}_{0}=\mathbb{E}\left[\left(\mathbf{z}_{0}-\widehat{\mathbf{z}}_{0}\right)\left(\mathbf{z}_{0}-\widehat{\mathbf{z}}_{0}\right)^{T}\right] \\
& \text { (4) while } k=1, \ldots, N_{t} \\
& \text { (5) Input prediction stage: } \\
& \text { (6) } \quad \widetilde{\mathbf{R}}_{k}=\mathbf{C}^{m} \mathbf{P}_{k}^{z-} \mathbf{C}^{m T}+\mathbf{R} \\
& \text { (7) } \quad \widehat{\mathbf{u}}_{k}=\mathbf{K}_{k}^{u}\left(\mathbf{y}_{k}-\mathbf{C}^{m} \overline{\mathbf{z}}_{k}^{-}\right) \\
& \text {(8) } \quad \mathbf{K}_{k}^{u}=\mathbf{P}_{k}^{u}\left[\begin{array}{ll}
\mathbf{D}^{m} & \mathbf{C}^{m} \mathbf{B}
\end{array}\right]^{T} \widetilde{\mathbf{R}}_{k}^{-1} \\
& \text { (9) } \quad \mathbf{P}_{k}^{u}=\left[\begin{array}{ll}
\mathbf{D}^{m} & \mathbf{C}^{m} \mathbf{B}
\end{array}\right]^{T} \widetilde{\mathbf{R}}_{k}^{-1}\left[\begin{array}{ll}
\mathbf{D}^{m} & \mathbf{C}^{m} \mathbf{B}
\end{array}\right]^{+} \\
& \text {(10) Measurement updating stage: } \\
& \text { (11) } \widehat{\mathbf{z}}_{k}^{+}=\overline{\mathbf{z}}_{k}^{+}+\mathbf{V}_{k} \widehat{\mathbf{u}}_{k} \\
& \text { (12) } \mathbf{P}_{k}^{z+}=\mathbf{P}_{k}^{\bar{z}+}+\mathbf{V}_{k} \mathbf{P}_{k}^{u} \mathbf{V}_{k}^{T} \\
& \text { (13) } \quad \mathbf{V}_{k}=\left[\begin{array}{ll}
\mathbf{0} & \mathbf{B}
\end{array}\right]-\mathbf{K}_{k}^{\bar{z}}\left[\begin{array}{ll}
\mathbf{D}^{m} & \mathbf{C}^{m} \mathbf{B}
\end{array}\right] \\
& \text { (14) Time updating stage: } \\
& \text { (15) } \quad \overline{\mathbf{z}}_{k}^{-}=\mathbf{A} \widehat{\mathbf{z}}_{k-1}^{+}+\mathbf{B}\left(\mathbf{D}^{m+}\left[\begin{array}{ll}
\mathbf{D}^{m} & 0
\end{array}\right] \mathbf{K}_{k-1}^{u}\left(\mathbf{y}_{k}-\mathbf{C}^{m} \overline{\mathbf{z}_{k-1}}\right)\right) \\
& \text { (16) } \overline{\mathbf{z}}_{k}^{+}=\overline{\mathbf{z}}_{k}^{-}+\mathbf{K}_{k}^{\bar{z}}\left(\mathbf{y}_{k}-\mathbf{C}^{m} \overline{\mathbf{z}}_{k}^{-}\right) \\
& \text {(17) } \mathbf{P}_{k}^{\bar{z}-}=\mathbf{A P}_{k-1}^{z+} \mathbf{A}^{T}+\mathbf{Q}_{z} \\
& \text { (18) } \quad \mathbf{K}_{\underline{k}}^{\bar{z}}=\mathbf{P}_{k}^{\bar{z}-} \mathbf{C}^{m T} \widetilde{R}_{k}^{-1} \\
& \text { (19) } \quad \mathbf{P}_{k}^{\bar{z}+}=\left(\mathbf{I}-\mathbf{K}_{k}^{\bar{z}} \mathbf{C}^{m}\right) \mathbf{P}_{k}^{\bar{z}-} \\
& \widehat{\mathbf{y}}_{k}=\mathbf{C} \widehat{\mathbf{z}}_{k}^{+}+\mathbf{D} \widehat{\mathbf{u}}_{k} \\
& \text { (21) end while } \\
& \text { (22) Obtain } \widehat{\mathbf{y}}_{k}, \widehat{\mathbf{z}}_{k}^{+} \text {and } \widehat{\mathbf{u}}_{k} \text {. }
\end{aligned}
$$

Algorithm 2: RTSKF.

through the unbiased estimation of state vector $\overline{\mathbf{z}}_{k}^{+}$obtained by the two filters. If the outputs are fully measured, the filter deals with a denoising problem; if the measurements are incomplete, then the filter deals with a denoising problem and an estimation problem. And by changing the form of matrix $\mathbf{C}$, different types of response can be obtained. The EIKF is used in the proposed method.

2.3. Displacement Field Estimation by Modal Method. In this study, a modal method is used for the construction of the 
displacement-strain-transformation relationship. It is possible to represent the dynamic displacements $\omega$ and strains $\varepsilon$ by multiplying these shape functions with weighting factors or modal coordinates $\mathbf{q}$ using displacement mode shapes $\boldsymbol{\Phi}$ and strain mode shapes $\Psi$ :

$$
\begin{aligned}
\{\boldsymbol{\omega}\}_{n_{d}} & =\{\boldsymbol{\Phi}\}_{n_{d} \times m} \cdot\{\mathbf{q}\}_{m}, \\
\{\boldsymbol{\varepsilon}\}_{n_{e}} & =\{\boldsymbol{\Psi}\}_{n_{e} \times m} \cdot\{\mathbf{q}\}_{m},
\end{aligned}
$$

where $n_{d}$ denotes the number of DOFs and $n_{e}$ denotes the number of elements, and $\Phi$ can be formed as

$$
\boldsymbol{\Phi}=\left[\begin{array}{llll}
\phi_{1} & \phi_{2} & \cdots & \phi_{m}
\end{array}\right]=\left[\begin{array}{cccc}
\phi_{11} & \phi_{21} & \cdots & \phi_{m 1} \\
\phi_{12} & \phi_{22} & \cdots & \phi_{m 2} \\
\cdots & \cdots & \cdots & \cdots \\
\phi_{1 n_{d}} & \phi_{2 n_{d}} & \cdots & \phi_{m n_{d}}
\end{array}\right] .
$$

$\Psi$ can be transformed from $\Phi$ :

$$
\boldsymbol{\Psi}=\mathbf{T} \boldsymbol{\Phi}=\left[\begin{array}{llll}
\psi_{1} & \psi_{2} & \cdots & \psi_{m}
\end{array}\right]=\left[\begin{array}{cccc}
\psi_{11} & \psi_{21} & \cdots & \psi_{m 1} \\
\psi_{12} & \psi_{22} & \cdots & \psi_{m 2} \\
\cdots & \cdots & \cdots & \cdots \\
\psi_{1 n_{e}} & \psi_{2 n_{e}} & \cdots & \psi_{m n_{e}}
\end{array}\right]
$$

where $\mathbf{T}$ denotes the strain-displacement matrix, which is related to the shape function of the element.

Displacement mode shapes $\Phi$ can be extracted from modal analysis of finite element model (FEM) or obtained by undamped eigenvalue analysis using equation (2).

When the number of sensors $n_{d}$ is larger than the number of selected modes $m$, the least square solution of $\{\mathbf{q}\}_{m}$ is

$$
\{\mathbf{q}\}_{m}=\{\boldsymbol{\Psi}\}_{n_{e} \times m}^{+} \boldsymbol{\varepsilon}_{n_{e}},
$$

where $\{\boldsymbol{\Psi}\}_{n_{e} \times m}^{+}$denotes the pseudoinverse of $\{\boldsymbol{\Psi}\}_{n_{d} \times m}$, which satisfies $\quad\left\{\boldsymbol{\Psi}_{n_{e} \times m}^{+}=\left(\{\boldsymbol{\Psi}\}_{n_{e} \times m}^{T}\{\boldsymbol{\Psi}\}_{n_{e} \times m}\right)^{-1}\{\boldsymbol{\Psi}\}_{n_{e} \times m}^{T}\right.$. Estimated displacement $\widehat{\boldsymbol{\omega}}_{n_{d}}$ can be inferred based on equations (11) and (15):

$$
\widehat{\boldsymbol{\omega}}_{n_{d}}=\{\boldsymbol{\Phi}\}_{n_{d} \times m}\{\boldsymbol{\Psi}\}_{n_{e} \times m}^{+} \boldsymbol{\varepsilon}_{n_{e}} .
$$

\section{Modal-Based Kalman Filter Approach for Structure Response Reconstruction}

3.1. General Framework. The displacement and velocity response can be reconstructed by introducing equations (8) and (9) into the frame of Kalman filters (Algorithms 1 and 2). However, the measurements of displacement and velocity are also required to obtain a better reconstruction precision. The filter via only acceleration measurements causes drift effect and a poor reconstruction result of displacement and excitation.

To avoid the above problems, the strain measurements are acquired in the proposed approach. Then, instead of equation (8), matrix $\mathbf{C}$ can be reformed:

$$
\mathbf{C}=\left[\begin{array}{cc}
\boldsymbol{\Psi} & \mathbf{0} \\
\mathbf{0} & \boldsymbol{\Phi} \\
-\boldsymbol{\Phi} \omega_{0}^{2} & -2 \Phi \xi \omega_{0}
\end{array}\right]
$$

Here, a modal-based EIKF approach (M-EIKF) which can obtain the full strain, displacement, velocity, acceleration, and excitation is proposed:

Step 1. Acquire strain, velocity, acceleration, and excitation of the structure via Algorithm 1 using strain and acceleration measurements;

Step 2. Acquire displacement via equation (16) using the reconstructed strain response.

This approach is a two-stage framework which combines EIKF and modal method. The change of the $\mathbf{C}$ matrix does not affect the essence of the EIKF algorithm. The block diagram of the proposed approach is shown in Figure 1.

3.2. Optimal Sensor Placement. To determine optimal sensor placement for response reconstruction using Algorithm 1, the objective function should be determined first. Considering the steady-state error covariance for the best response reconstruction, the precision of reconstruction can be expressed as $\boldsymbol{\delta}_{k}$ which is defined as follows:

$$
\boldsymbol{\delta}_{k}=\mathbf{y}_{k}-\widehat{\mathbf{y}}_{k}=\left(\mathbf{C} \mathbf{z}_{k}+\mathbf{D} \mathbf{u}_{k}\right)-\left(\mathbf{C} \widehat{\mathbf{z}}_{k}^{+}+\mathbf{D} \widehat{\mathbf{u}}_{k}\right) .
$$

The covariance matrix of reconstruction error is

$$
\boldsymbol{\Delta}_{k}=E\left[\begin{array}{l}
\boldsymbol{\delta}_{k} \boldsymbol{\delta}_{k}^{T}
\end{array}\right]=\left[\begin{array}{ll}
\mathbf{C} & \mathbf{D}
\end{array}\right]\left[\begin{array}{cc}
\mathbf{P}_{k}^{z+} & \mathbf{P}_{k}^{z u} \\
\mathbf{P}_{k}^{u z} & \mathbf{P}_{k}^{u}
\end{array}\right]\left[\begin{array}{ll}
\mathbf{C}^{T} & \mathbf{D}^{T}
\end{array}\right] .
$$

$\Delta_{k}$ can converge to a fixed value $\widetilde{\Delta}$ as filter is working:

$$
\lim _{k \longrightarrow \infty} \boldsymbol{\delta}_{k}=\tilde{\Delta} \text {. }
$$

The objective function can be expressed as

$$
\operatorname{Obj}=\min (\operatorname{trace}(\widetilde{\Delta})) \text {. }
$$

The placement method should be determined since the objective function is defined. Here, backward sequential sensor placement (BSSP) is used to obtain the best location of the sensors. BSSP (Algorithm 3) is used in an inverse order, starting with sensors placed at all DOFs of the structure and removing successively one sensor at a time from the location that results in the smallest increase in the objective function. Only strain gauges and acceleration sensors are chosen in the process of BSSP to confirm that displacement and velocity will be not measured.

\section{Numerical Example}

4.1. Descriptions of Truss. To verify the performance of the proposed method, a 25-DOF truss structure (in Figure 2) is adopted, which has 14 nodes and 25 elements. Each node from 2 nd to 13 th has two DOFs: a vertical DOF and a 




Figure 1: Block diagram of M-EIKF.

(1) Initialization: All sensors selected, number of sensors $n_{d}$ and sensor configuration $L_{n_{d}}$;

(2) while Number of sensors $n_{d}>$ number of excitations $n$ do

(3) $n_{d}=n_{d}-1$;

(4) for $i=1$ to $n_{d}$ do

(5) Obtain configuration $L_{n_{d}}^{i}$ by removing sensor $i$;

(6) Obtain the Obj of configuration $L_{n_{d}}^{i}$ via Algorithm 1;

(7) end for

(8) Select the sensor configuration $L_{n_{d}}^{i}$ that minimizes the Obj as the new $L_{n_{d}}$;

(9) end while

(10) Obtain $L_{n_{d}}$.

Algorithm 3: BSSP.

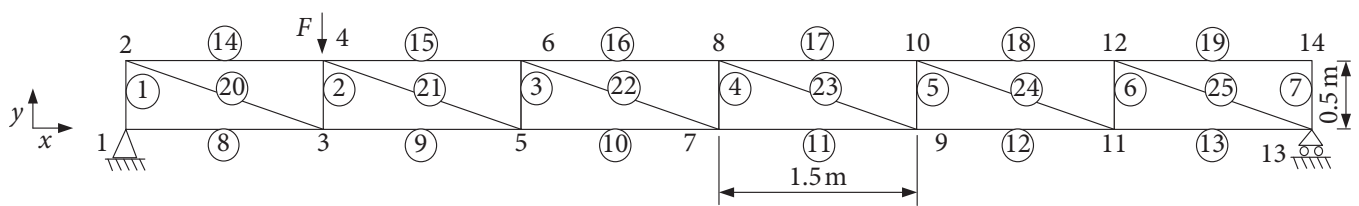

Figure 2: Two-dimensional truss structure.

horizontal DOF. The 14th node has a horizontal DOF. Rayleigh damping $(\mathbf{C}=\alpha \mathbf{M}+\beta \mathbf{K})$ is adopted to the model, in which $\alpha$ is 12.57 and $\beta$ is $1.69 \times 10^{-4}$. The mass and stiffness damping coefficients are defined through experience. Sparse matrix solver is used as the FEM solver. A random excitation and an impulsive excitation are vertically applied on node 4, respectively. The details of the applied forces are shown in Figure 3. Acceleration sensors are applied at each DOF and strain gauges are applied at each element.

4.2. Optimal Sensor Placement. Using BSSP, the convergence curve of Obj is shown in Figure 4. And the best configuration of sensors is obtained, which is shown in Figure 5. The configuration of 5 sensors is listed in Table 1, which is used in Section 4.3 for reconstruction of response and excitation.

In this approach, strain gauge and acceleration sensor are optimized at the same time, so horizontal ordinate denotes the total numbers of strain gauges and acceleration sensors in Figures 4 and 5. As can be seen in Figure 4, the value of Obj increases while the number of sensors becomes smaller. The objective function reaches a relatively small value when the number of sensors is 5 , which is chosen as the final configuration. When the number of sensors is over 20, the reconstruction error becomes very small. The sensor configuration could be changeable, and the Obj curve cannot 


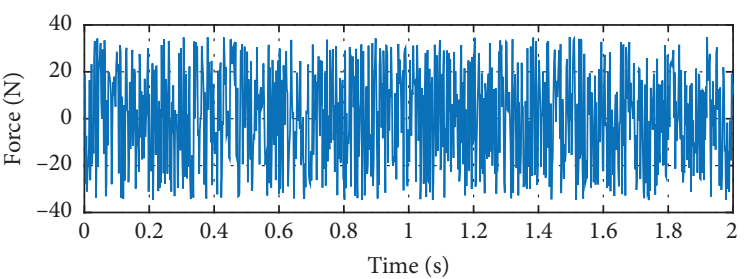

(a)

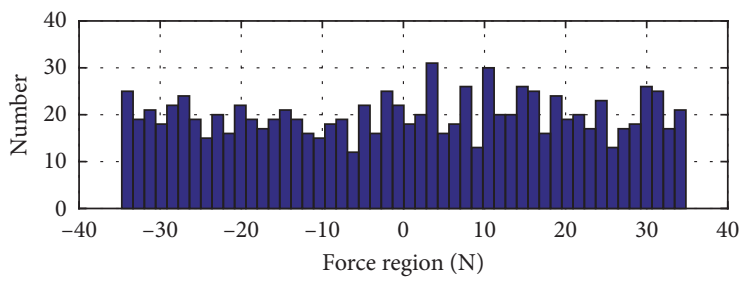

(c)

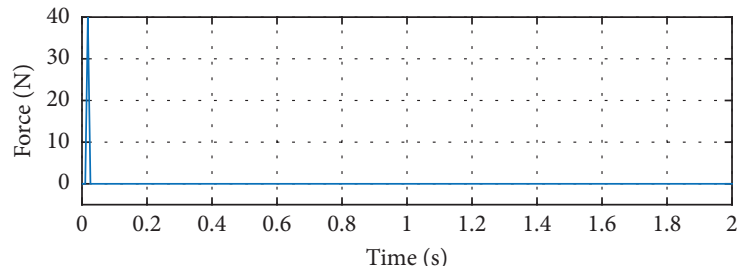

(b)

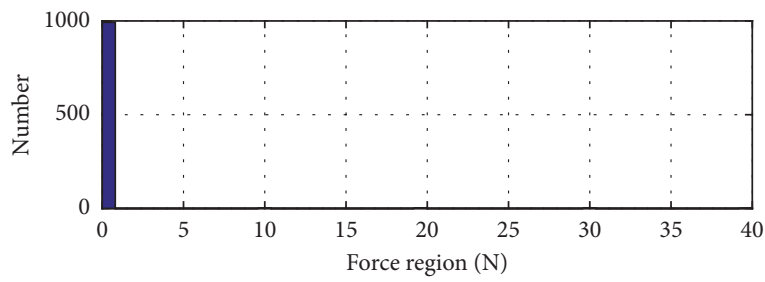

(d)

FIGURE 3: Applied forces. (a) Time history of random excitation and (c) its histogram; (b) time history of impulsive excitation and (d) its histogram.



Figure 4: Obj curve for truss structure.

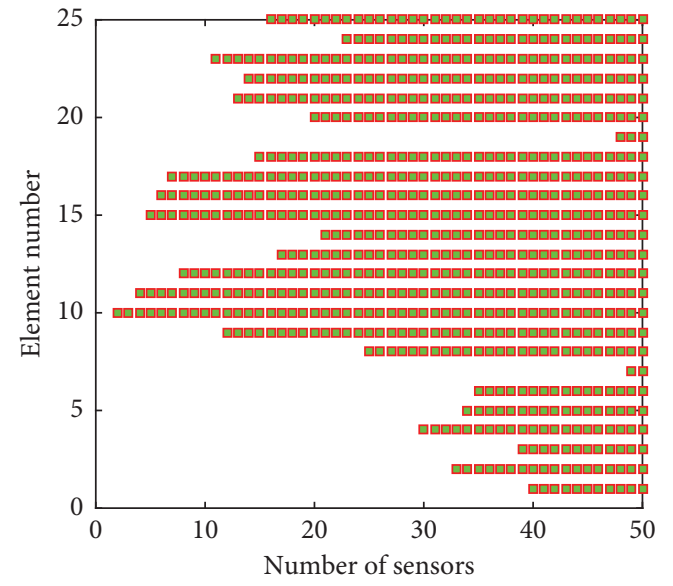

(a)

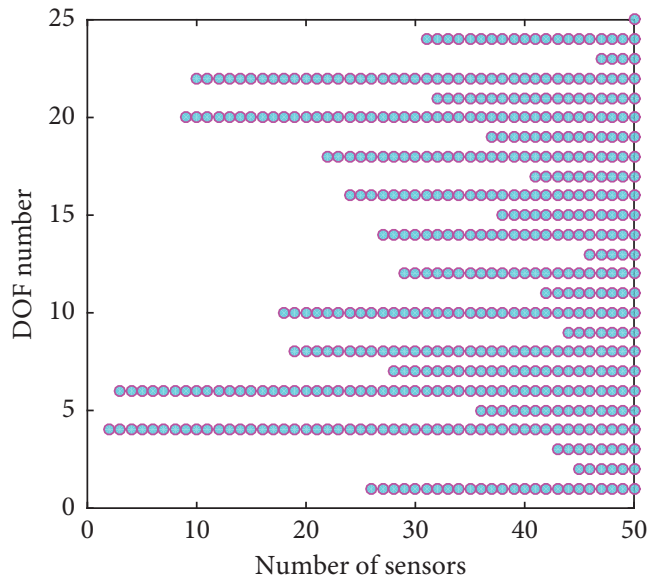

(b)

Figure 5: Configuration of sensors for truss structure. (a) Configuration of strain gauges and (b) configuration of acceleration sensors. 
TABLE 1: Sensor configuration by backward sequential method for truss structure (5 sensors).

\begin{tabular}{|c|c|}
\hline Sensor type & Location \\
\hline Strain gauges & $10-e, 11-e, 15-e$ \\
\hline Acceleration sensors & $3-Y, 4-Y$ \\
\hline
\end{tabular}

converge if RTSKF is used in the approach. As can be seen in Figure 5, it is interesting to see that acceleration sensors are applied at $Y$-direction preferentially (the even numbers of DOFs), which conforms to the mechanical properties of truss structure; because the strain of vertical bars of the truss is comparatively small, strain gauges are preferentially applied at elements with a number larger than 8 , which are crossbars and diagonal bars of the structure.

\subsection{Response Reconstruction and Excitation Estimation.} As for M-EIKF, the configuration in Table 1 is used for reconstruction while full acceleration measurements (accelerations of all DOFs are measured) are applied in EIKF and RTSKF, which are used for comparison.

The modeling error is set to $\mathbf{Q}_{z}=10^{-18} \times \mathbf{I}$, where $\mathbf{I}$ is an identity matrix. For M-EIKF, the measurement noise amplitude is assigned $2 \times 10^{-7} \mathrm{~m} / \mathrm{m}$, and $0.3 \mathrm{~m} / \mathrm{s}^{2}$ for strain and acceleration, respectively. For EIKF and RTSKF, the noise amplitude of acceleration measurement is assigned $0.3 \mathrm{~m} / \mathrm{s}^{2}$. Reconstructed responses and excitations under random and impulsive excitation are shown in Figures 6 and 7, respectively. The responses calculated by Newmark algorithm are used as the real value.

As can be seen from Figures 6 and 7, the drift effect, which is observed in the reconstruction of EIKF, does not exist in the estimations of M-EIKF, and it always happens in the reconstruction process of excitation and displacement for EIKF and RTSKF. RTSKF obtains a worse effect under both random and impulsive excitation than EIKF, especially for excitation and displacement. However, the reconstructed accelerations and velocities are in outstanding agreement with the real ones for all algorithms. Besides, the strain responses reconstructed by M-EIKF are also in great agreement with the theoretical value.

To confirm that the reconstruction of all DOFs can reach a great precision, root mean square errors (RMSEs) of all response types under random and impulsive excitation are shown in Figures 8 and 9, respectively. Considering the large error obtained by RTSKF, only EIKF and M-EIKF are plotted. As can be seen the displacement reconstruction of all DOFs for M-EIKF achieves great precision, which can also be seen from Figures 6 and 7. Both the velocity and acceleration reconstructed by the two methods are acceptable. Considering only 2 acceleration sensors are used in M-EIKF while the full acceleration measurements are applied in EIKF, it is reasonable that the acceleration curves of M-EIKF are slightly higher than those of EIKF. It is also interesting to see that the response on DOF and element of even numbers reaches larger RMSE value than that of odd numbers.

\section{Experimental Example}

5.1. Descriptions of Cantilever Beam. To further verify the validity of the proposed method, an experimental example of cantilever beam is used in this section. The material of the beam is ordinary steel with a Young's modulus of $128 \mathrm{GPa}$ and a density of $6440 \mathrm{~kg} / \mathrm{m}^{3}$, averagely. The beam is $600 \mathrm{~mm}$ long, $50 \mathrm{~mm}$ wide, and $7.8 \mathrm{~mm}$ thick. The beam is divided into 12 equal segments in the FEM, which contains 13 nodes, 12 elements, and 24 DOFs. Each node from 2nd to 13th has two DOFs: a rotational DOF and a vertical DOF. Considering that it is not easy to measure the rotational responses, the rotational DOFs are eliminated when deciding the sensor placement. The mass and stiffness damping coefficients of the beam are defined through FEM updating method. Figure 10 shows the picture of the beam, and Figure 11 shows its FEM. An impulsive excitation is applied at the 9th node vertically. For the numerical example, the covariance of the model error is used to verify the validity of the proposed method; for the experimental example, the FEM carries model error itself, so the manual set of covariance is not necessary.

5.2. Optimal Sensor Placement. Using BSSP, the convergence curve of Obj is shown in Figure 12. And the best configuration of sensors is obtained, which is shown in Figure 13. The configuration of 4 sensors is listed in Table 2, which is used in Section 5.3 for reconstruction of response and excitation.

As can be seen in Figure 12, the value of Obj increases while number of sensors becomes smaller, which is not as small as in Figure 4 because every rotational DOF is eliminated. As can be seen in Figure 13, the number of acceleration sensors is larger than the number of strain gauges when the number of sensors is below 15 . Nonetheless, one acceleration sensor and one strain gauge are chosen when the number of sensors is 2 , which indicates the necessity of multitype sensor placement. For the cantilever beam, the strain response near the fixed end is relatively large, and there is little response near the free end. Accordingly, the 1st and 5th elements are chosen for applying strain gauges preferentially, and the 11th and 12th elements are chosen lastly; on the contrary, the deformation of the free end is relatively larger than that of the fixed end, which is the same situation with acceleration response; hence, acceleration sensors are applied near the free end preferentially.

\subsection{Response Reconstruction and Excitation Estimation.} Sensor configuration in Table 2 is used in actual measurement. The ICP acceleration sensors are used; the type of strain gauges is BE120-3AA, attached with the strain regulator, and the connection type is $1 / 4$ bridge; network distributed acquisition instrument from China Orient Institute of Noise \& Vibration (COINV) is used for signal acquisition. 

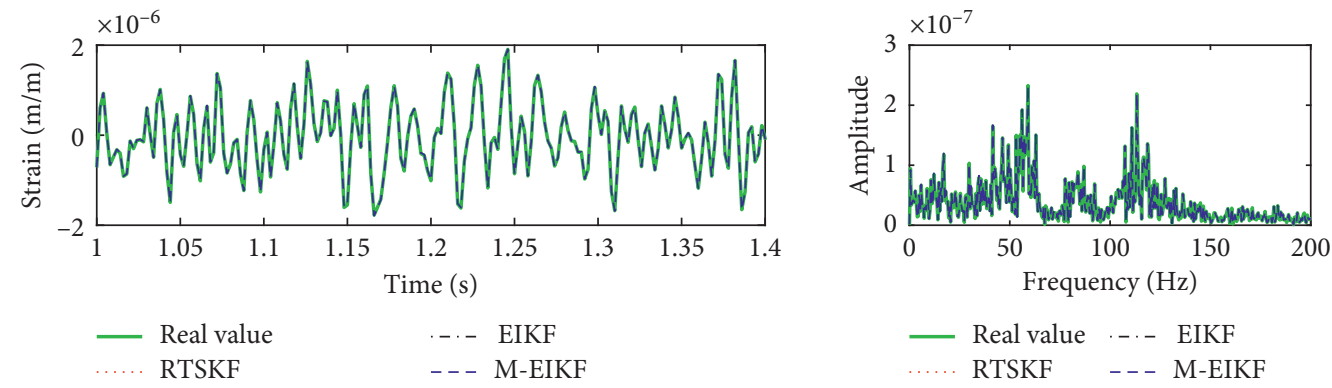

(a)
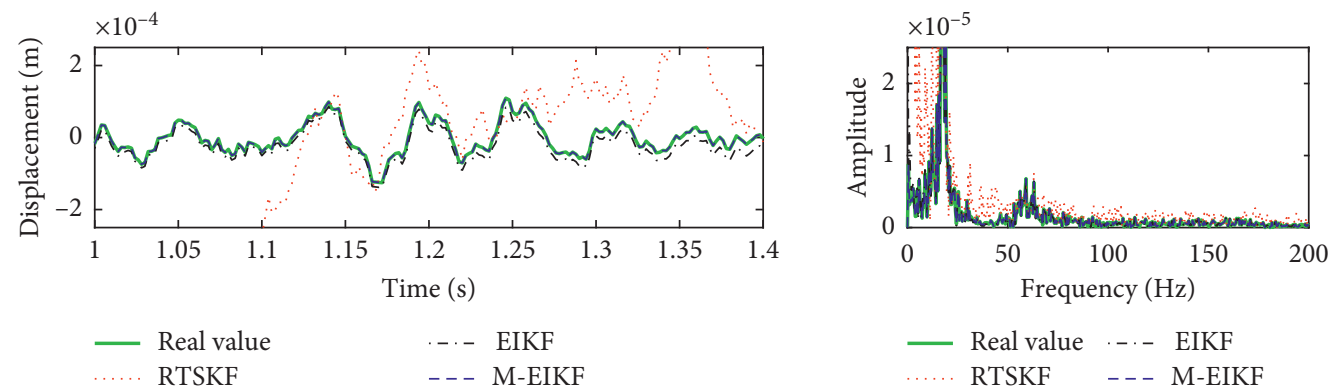

(b)
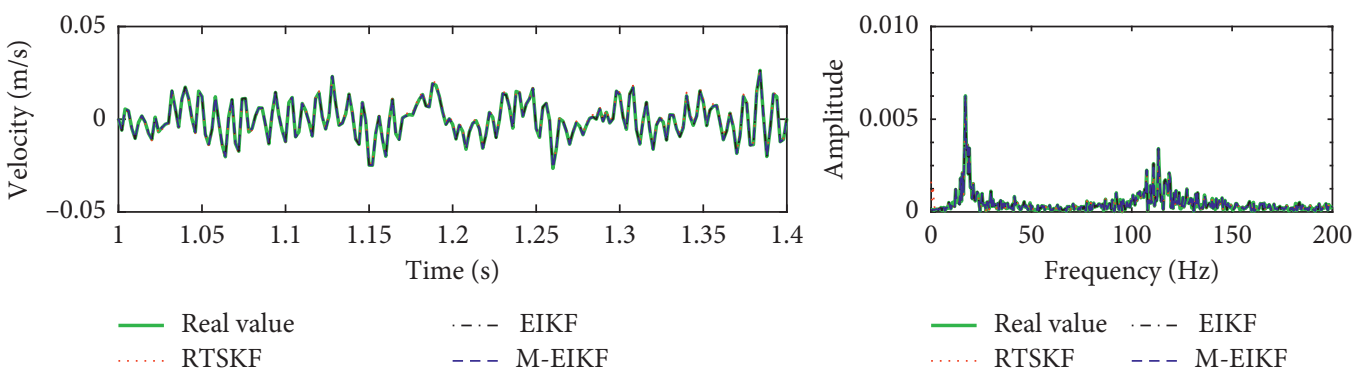

(c)
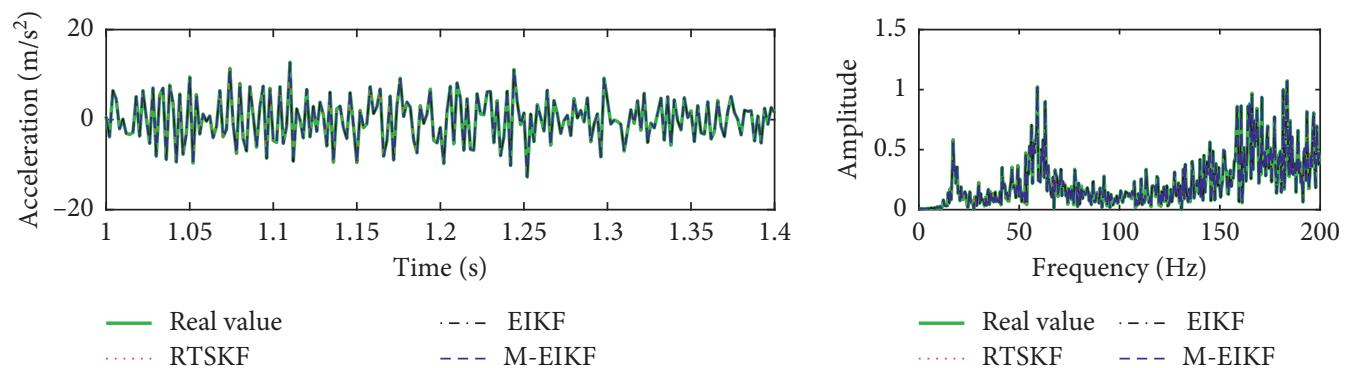

(d)
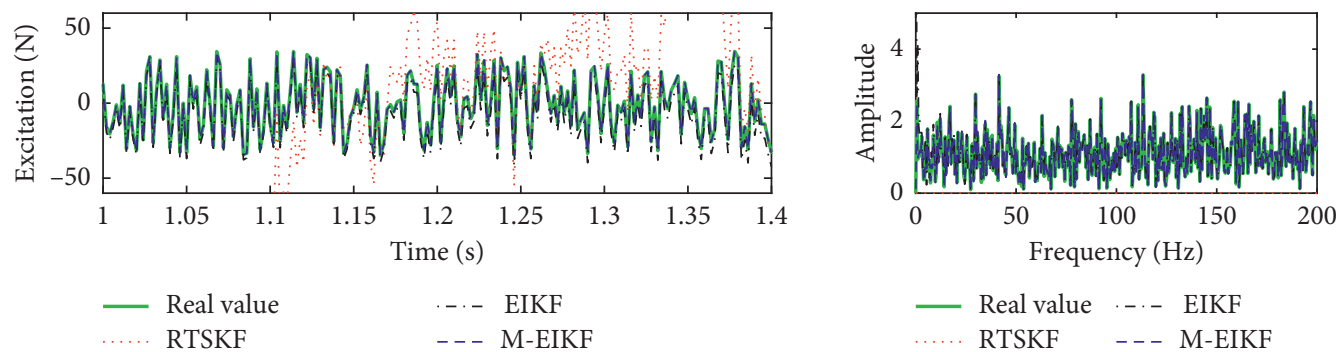

(e)

FIGURE 6: Reconstructed responses and excitation under random excitation for truss structure. (a) Time history of strain at 8th element and its amplitude spectrum; (b) time history of $Y$-direction displacement at 5th node and its amplitude spectrum; (c) time history of $Y$-direction velocity at 7th node and its amplitude spectrum; (d) time history of $Y$-direction acceleration at 9th node and its amplitude spectrum; (e) time history of random excitation and its amplitude spectrum. 

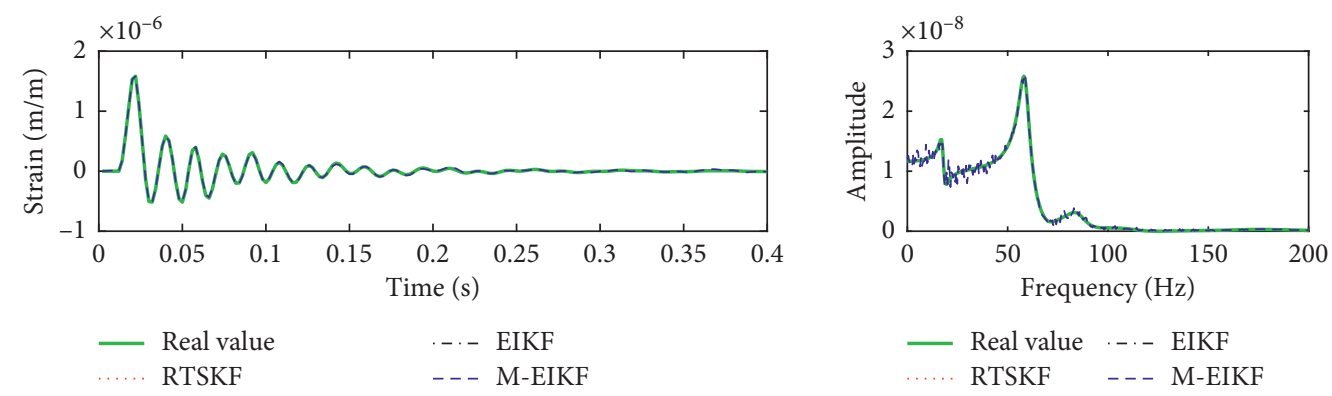

(a)
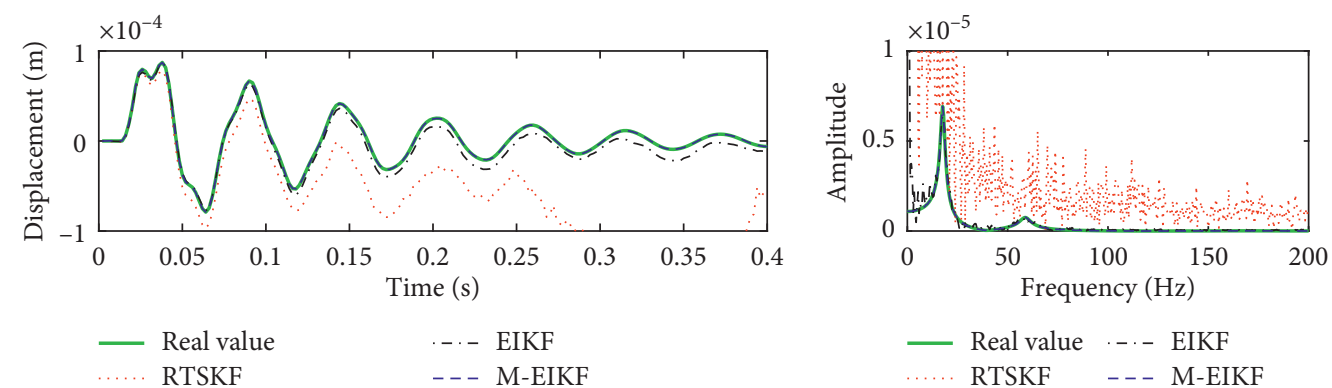

(b)
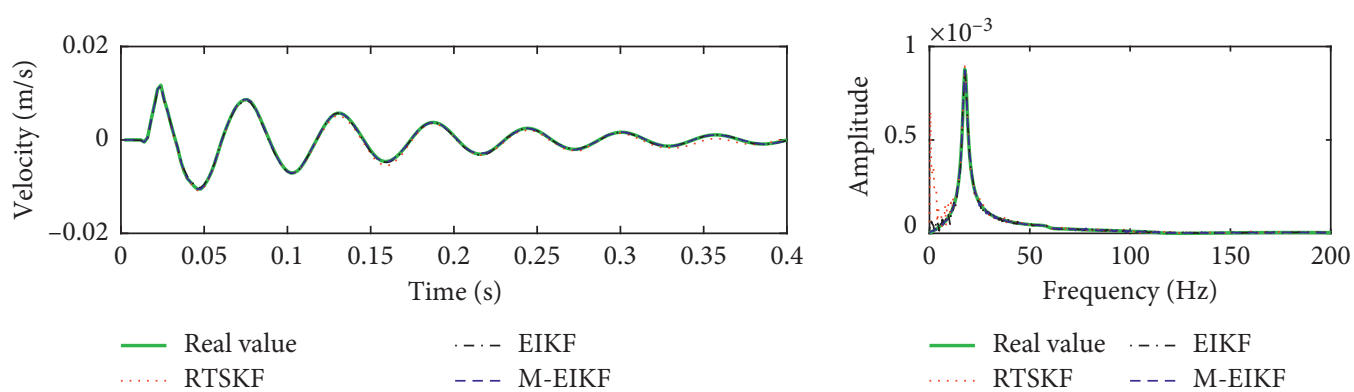

(c)
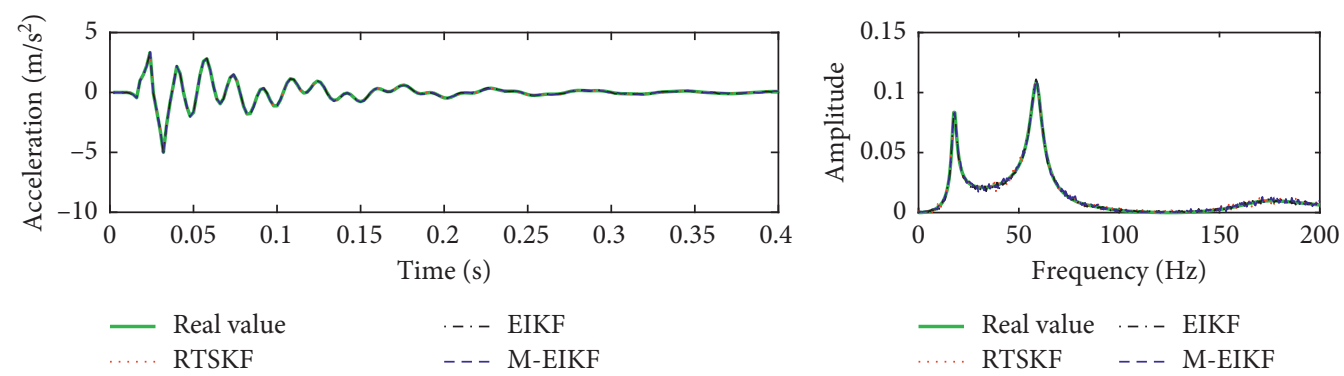

(d)
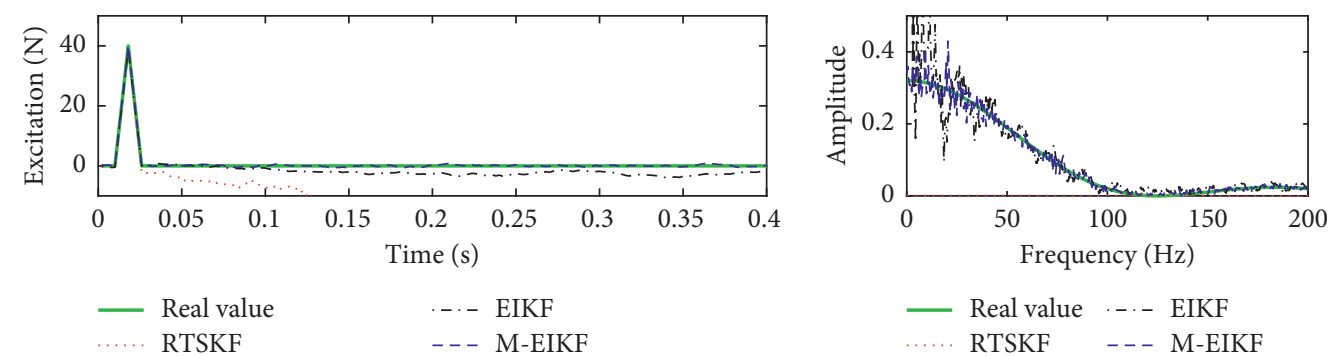

(e)

Figure 7: Reconstructed responses and excitation under impulsive excitation for truss structure. (a) Time history of strain at 8th element and its amplitude spectrum; (b) time history of $Y$-direction displacement at 5th node and its amplitude spectrum; (c) time history of $Y$ direction velocity at 7 th node and its amplitude spectrum; (d) time history of $Y$-direction acceleration at 9 th node and its amplitude spectrum; (e) time history of impulsive excitation and its amplitude spectrum. 




$$
\begin{aligned}
& - \text { EIKF } \\
& + \text { M-EIKF }
\end{aligned}
$$

(a)

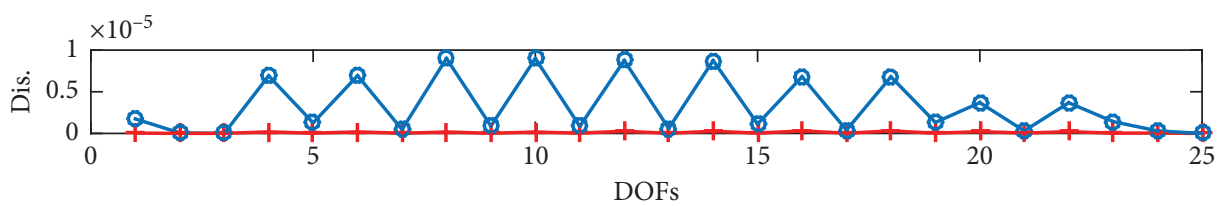

- EIKF
+ M-EIKF

(b)



- 0 - EIKF

+ M-EIKF

(c)

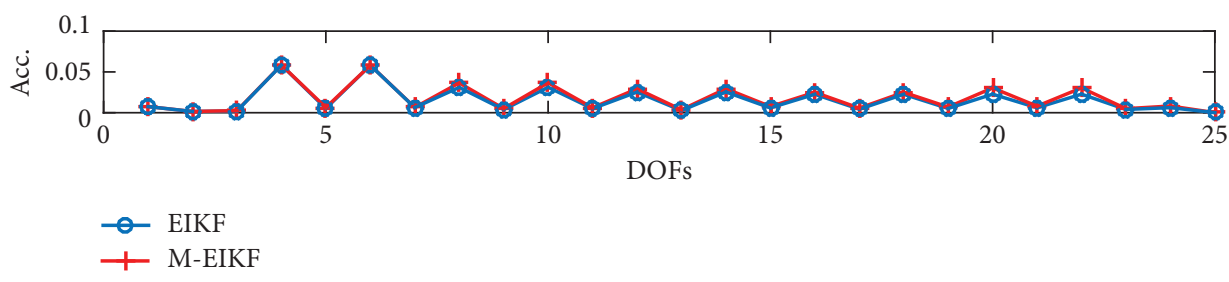

(d)

FIGURE 8: RMSEs of all DOFs under random excitation for truss structure. (a) RMSEs of reconstructed strain; (b) RMSEs of reconstructed displacement; (c) RMSEs of reconstructed velocity; (d) RMSEs of reconstructed acceleration.

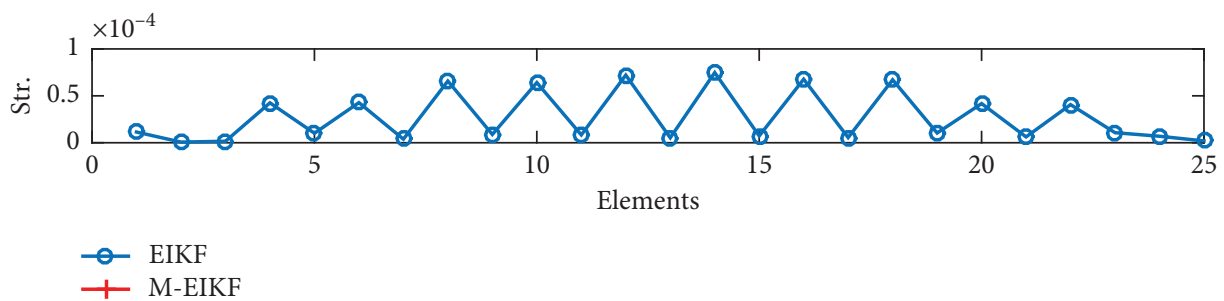

(a)

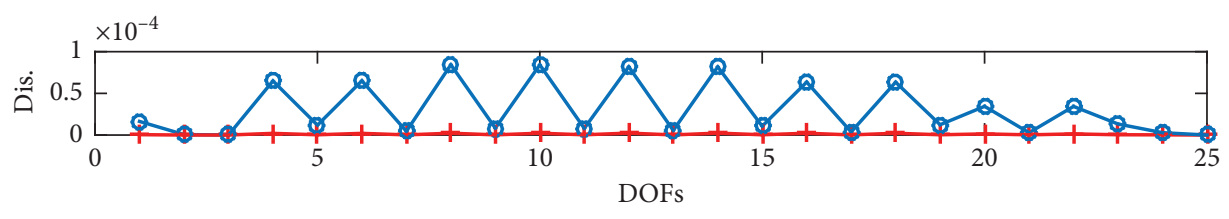

- - EIKF

+ M-EIKF

(b)

Figure 9: Continued. 




(c)

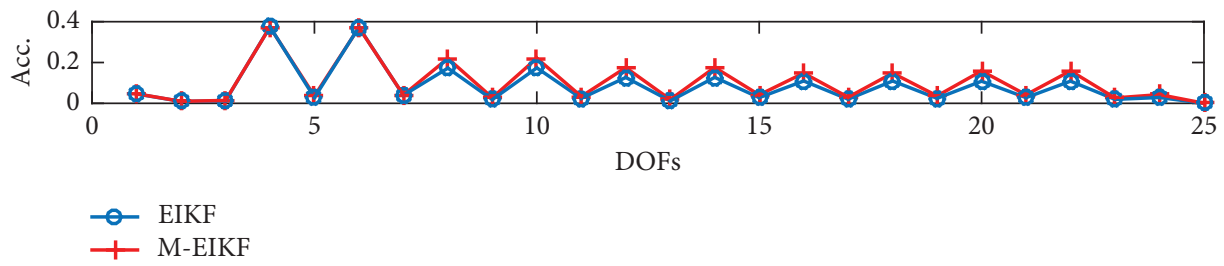

(d)

FIGURE 9: RMSEs of all DOFs under impulsive excitation for truss structure. (a) RMSEs of reconstructed strain; (b) RMSEs of reconstructed displacement; (c) RMSEs of reconstructed velocity; (d) RMSEs of reconstructed acceleration.

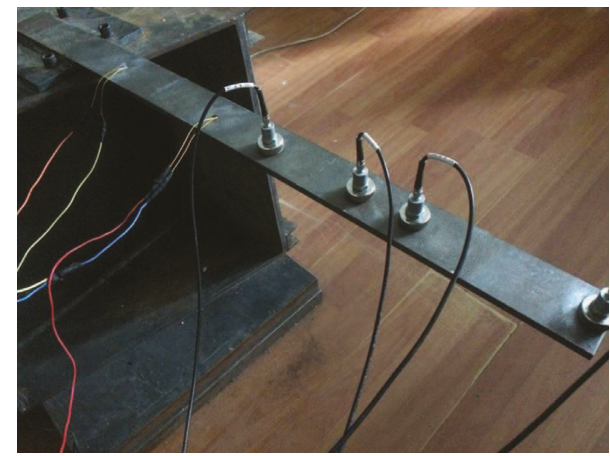

FIGURE 10: Cantilever beam.

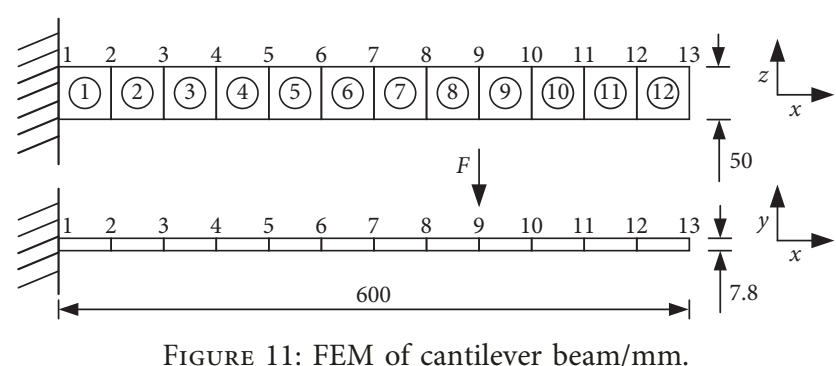

Configuration of actual sensor placement is shown in Figure 14 . The $Y$-direction acceleration at 7 th node and the strain at 2 nd element are collected for comparison with the reconstructed signals. Figure 15 gives the details of equipment connection.

The responses of part of the beam are shown in Figure 16. For the reason that the displacement and velocity response are hard to measure, velocity is eliminated and the simulative displacement signal by Newmark algorithm (theoretical value) is used. As can be seen in Figures 16(a) and $16(c)$, reasonable estimates of strain and acceleration of structures are accomplished for both frequency and

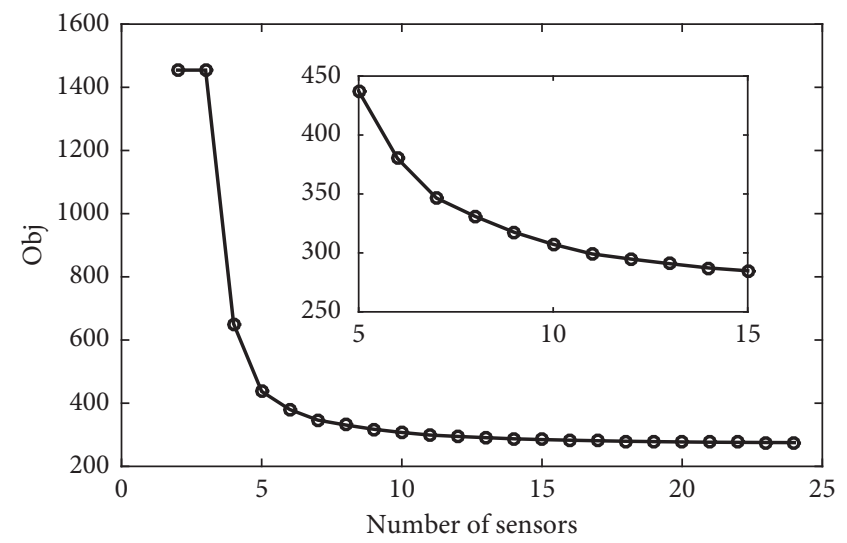

Figure 12: Obj curve for cantilever beam.

amplitude of the signal. In Figure 16(b), the difference between simulative signal and reconstructed signal is shown, in which the first-order frequency is slightly different. This difference is caused by model error, which makes Newmark algorithm simulate wrong signals with errors. And the drift effect of EIKF is still observed, which can also be found in Section 4.3. In Figure 16(d), a reasonable excitation signal is predicted using M-EIKF while EIKF gives a diverging one. Furthermore, the M-EIKF is not absolutely precise given the thought that material of the beam is uneven and the FEM is not precise enough.

\section{Conclusion}

In this study, a modal-based Kalman filter approach (MEIKF) for structure response reconstruction is proposed to provide optimal experimental design for structural response reconstruction. Considering the actual situation in engineering, the modal method is used to construct the displacement response of the structure, which avoids the direct measure of displacement response. The proposed method is 




(a)

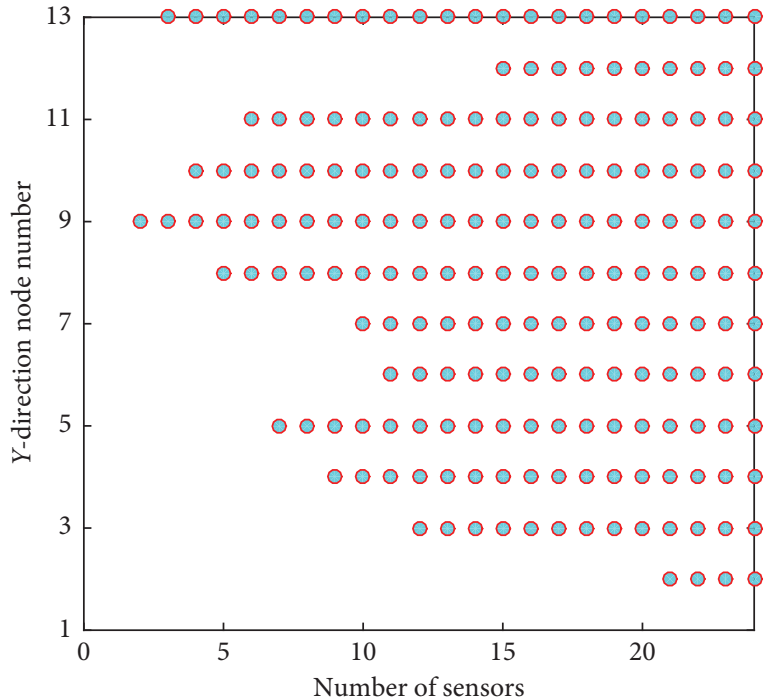

(b)

Figure 13: Configuration of sensors for cantilever beam. (a) Configuration of strain gauges and (b) configuration of acceleration sensors.

TABLE 2: Sensor configuration by backward sequential method for cantilever beam (4 sensors).

\begin{tabular}{lr}
\hline Sensor type & Location \\
\hline Strain gauges & $5-e$ \\
Acceleration sensors & $9-Y, 10-Y, 13-Y$ \\
\hline
\end{tabular}

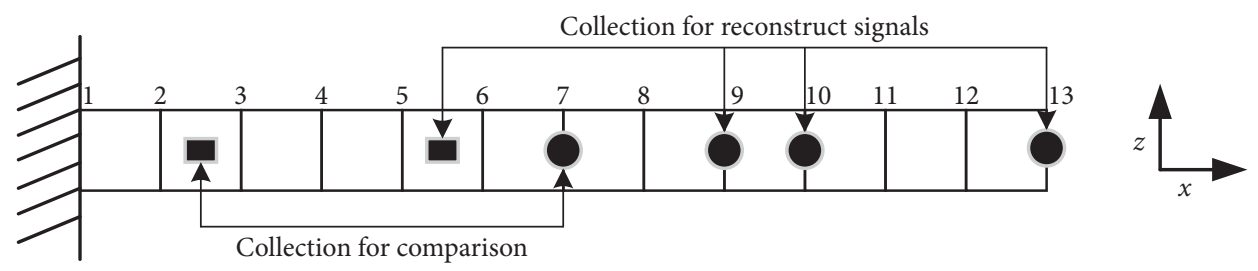

FIgURE 14: Configuration of actual sensor placement.
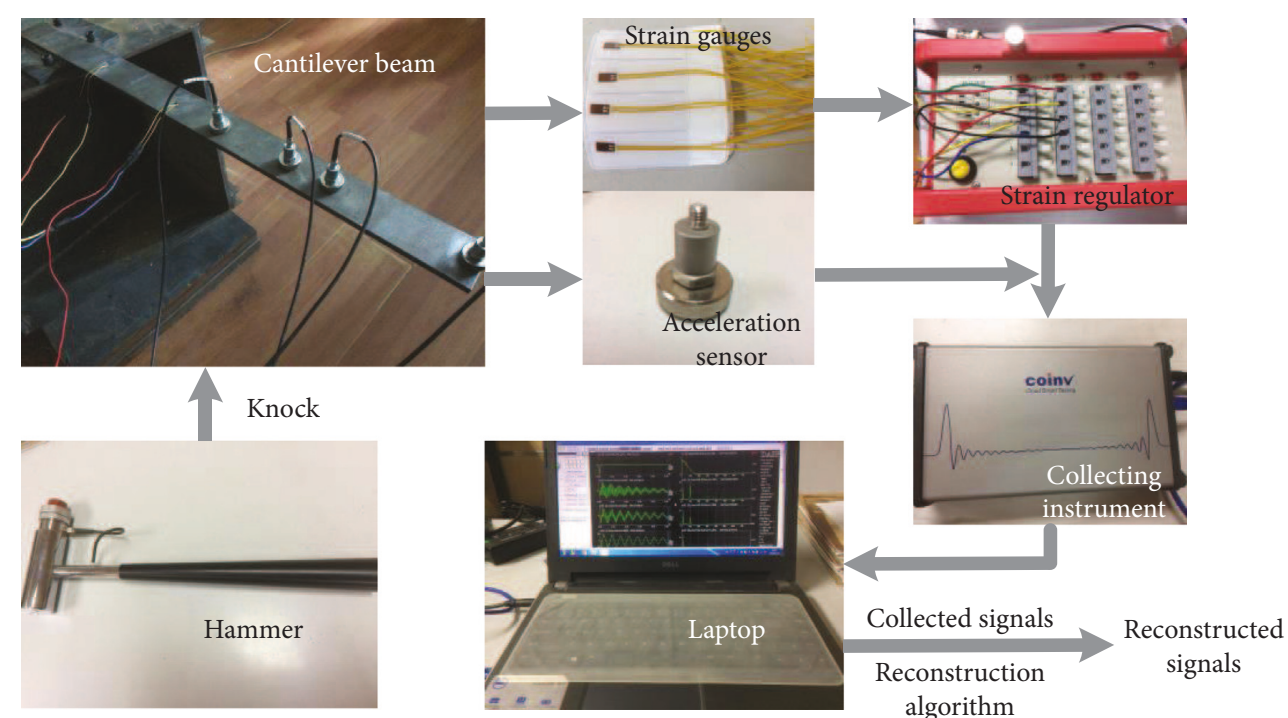

FIGURE 15: Equipment connection diagram. 

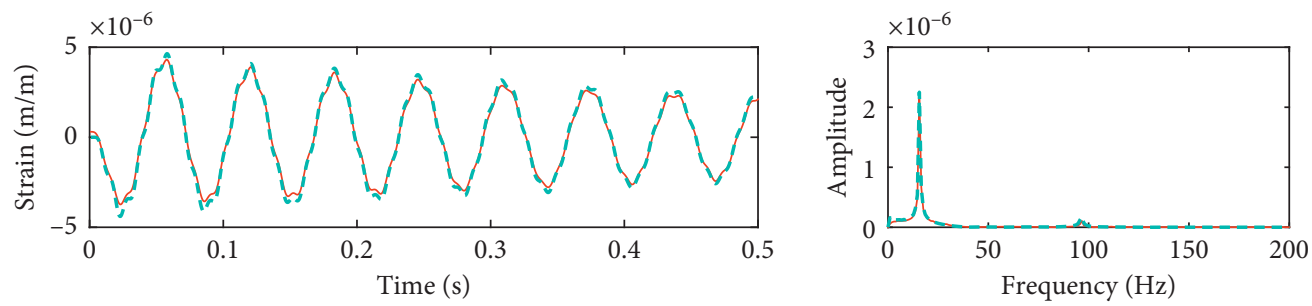
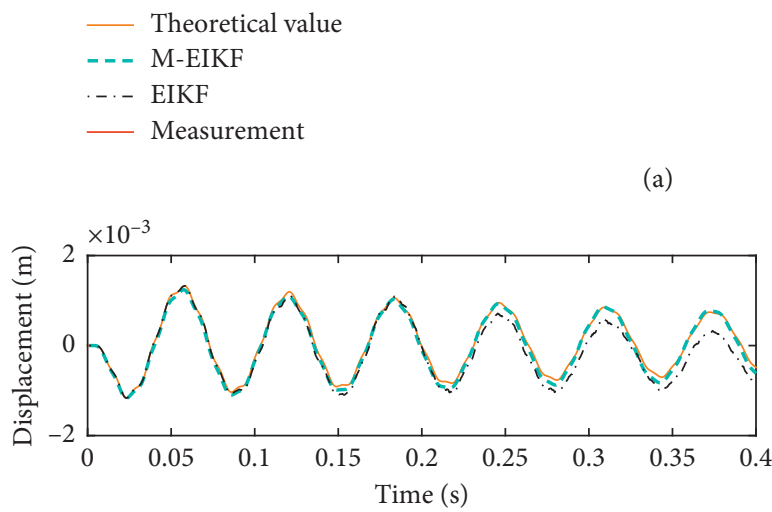

_ Theoretical value

- - - M-EIKF

... - EIKF

- Measurement

(b)

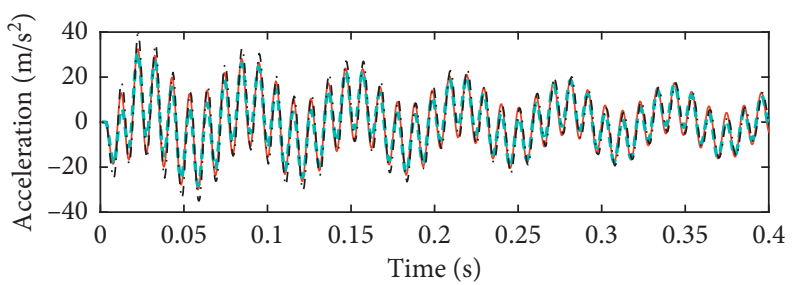

_ Theoretical value

- - - M-EIKF

...- EIKF

_ Measurement

(c)

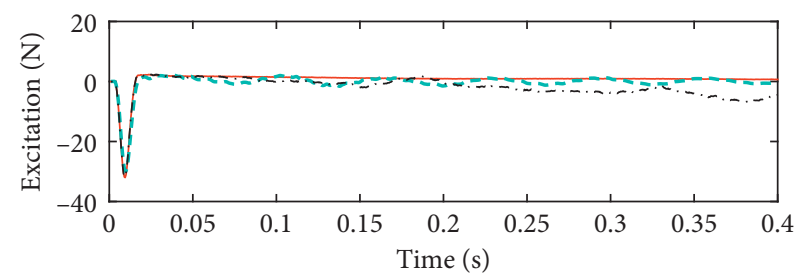

_ Theoretical value

- - - M-EIKF

... EIKF

_ Measurement

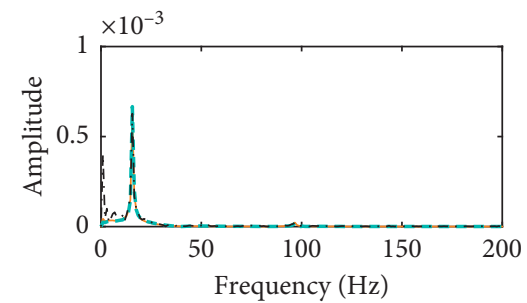

_ Theoretical value

- - - M-EIKF

.. - EIKF

_ Measurement

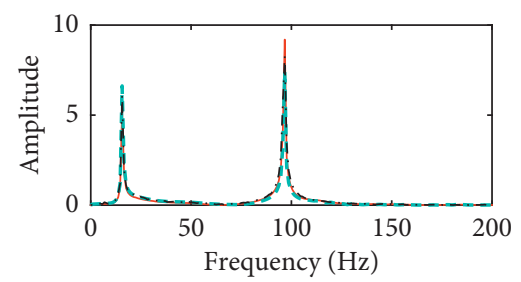

Theoretical value

- - - M-EIKF

...- EIKF

_ Measurement

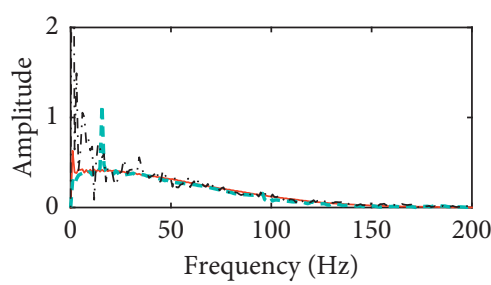

_ Theoretical value

- - - M-EIKF

... EIKF

_ Measurement

(d)

Figure 16: Reconstructed responses and excitation under impulsive excitation for truss structure. (a) Time history of strain at 2nd element and its amplitude spectrum; (b) time history of $Y$-direction displacement at 7th node and its amplitude spectrum; (c) time history of $Y$ direction acceleration at 7th node and its amplitude spectrum; (d) time history of impulsive excitation and its amplitude spectrum.

demonstrated by a numerical example of a two-dimensional structure under two different excitation types, and an experimental example of cantilever beam. Through an optimal sensor placement schedule, the final locations of the applied sensors are determined. Results show that M-EIKF gives better reconstructed precision of different response types 
and excitations and uses only two common types of sensors: acceleration sensor and strain gauge. Both the type and number of sensors obtained by M-EIKF are promising. The proposed method does not change the essence of EIKF algorithm, and it is a two-stage approach which can give a better solution than EIKF using strain mode shapes. Due to that strain mode shapes can easily be obtained using displacement mode shapes, the proposed method can be an easy-implement methodology for structural health monitoring.

\section{Data Availability}

The data used to support the findings of this study are available from the corresponding author upon request.

\section{Conflicts of Interest}

The authors declare that they have no conflicts of interest.

\section{Acknowledgments}

This research is supported by National Natural Science Foundation of China (Nos. 61463028 and 51768035), Collaborative Innovation Team Project of Universities in Gansu Province, China (No. 2018C-12), Foundation of a Hundred Youth Talent Training Program of Lanzhou Jiaotong University (No. 152022), and Talent Project of Lanzhou City (No. 2017-RC-66).

\section{References}

[1] A. P. V. Urgueira, R. A. B. Almeida, and N. M. M. Maia, "On the use of the transmissibility concept for the evaluation of frequency response functions," Mechanical Systems and Signal Processing, vol. 25, no. 3, pp. 940-951, 2011.

[2] W. Liu and D. J. Ewins, "Transmissibility properties of MDOF systems," Proceedings of SPIE-The International Society for Optical Engineering, vol. 5377, pp. 1528-1535, 1998.

[3] S. S. Law, J. Li, and Y. Ding, "Structural response reconstruction with transmissibility concept in frequency domain," Mechanical Systems and Signal Processing, vol. 25, no. 3, pp. 952-968, 2011.

[4] J. Li and S. S. Law, "Substructural response reconstruction in wavelet domain," Journal of Applied Mechanics, vol. 78, no. 4, pp. 925-948, 2011.

[5] J. He, X. Guan, and Y. Liu, "Structural response reconstruction based on empirical mode decomposition in time domain," Mechanical Systems and Signal Processing, vol. 28, no. 2, pp. 348-366, 2012.

[6] Z. Wan, S. Li, Q. Huang et al., "Structural response reconstruction based on the modal superposition method in the presence of closely spaced modes," Mechanical Systems \& Signal Processing, vol. 42, no. 1-2, pp. 14-30, 2014.

[7] X. H. Zhang, S. Zhu, Y. L. Xu, and X. J. Homg, "Integrated optimal placement of displacement transducers and strain gauges for better estimation of structural response," International Journal of Structural Stability and Dynamics, vol. 11, no. 3, pp. 581-602, 2011.

[8] C.-K. Ma, P.-C. Tuan, D.-C. Lin, and C.-S. Liu, "A study of an inverse method for the estimation of impulsive loads,"
International Journal of Systems Science, vol. 29, no. 6, pp. 663-672, 1998.

[9] K. Erazo and E. M. Hernandez, "A model-based observer for state and stress estimation in structural and mechanical systems: experimental validation," Mechanical Systems \& Signal Processing, vol. 43, no. 1-2, pp. 141-152, 2014.

[10] R. E. Kalman, "A new approach to linear filtering and prediction problems," Journal of Basic Engineering, vol. 82, no. 1, pp. 35-45, 1960.

[11] N. J. Gordon, D. J. Salmond, and A. F. M. Smith, "Novel approach to nonlinear/non-Gaussian Bayesian state estimation," IEE Proceedings F-Radar and Signal Processing, vol. 140, no. 2, pp. 107-113, 1993.

[12] S. Gillijns and B. De Moor, "Unbiased minimum-variance input and state estimation for linear discrete-time systems with direct feedthrough," Automatica, vol. 43, no. 5, pp. 934-937, 2007.

[13] C. D. Zhang and Y. L. Xu, "Optimal multi-type sensor placement for response and excitation reconstruction," Journal of Sound and Vibration, vol. 360, pp. 112-128, 2016.

[14] S. J. Julier and J. K. Uhlmann, "New extension of the Kalman filter to nonlinear systems," Proceedings of SPIE-The International Society for Optical Engineering, vol. 3068, 1997.

[15] S. Eftekhar Azam, A. Ghisi, and S. Mariani, "Parallelized sigma-point Kalman filtering for structural dynamics," Computers \& Structures, vol. 92-93, pp. 193-205, 2012.

[16] J. Ching, J. L. Beck, and K. A. Porter, "Bayesian state and parameter estimation of uncertain dynamical systems," Probabilistic Engineering Mechanics, vol. 21, no. 1, pp. 81-96, 2006.

[17] E. N. Chatzi and A. W. Smyth, "The unscented Kalman filter and particle filter methods for nonlinear structural system identification with non-collocated heterogeneous sensing," Structural Control \& Health Monitoring, vol. 16, no. 1, pp. 99-123, 2009.

[18] S. Eftekhar Azam, M. Bagherinia, and S. Mariani, "Stochastic system identification via particle and sigma-point Kalman filtering," Scientia Iranica, vol. 19, no. 4, pp. 982-991, 2012.

[19] D. E. Nasr and G. A. Saad, "Optimal sensor placement using a combined genetic algorithm-ensemble Kalman filter framework," ASCE-ASME Journal of Risk and Uncertainty in Engineering Systems, Part A: Civil Engineering, vol. 3, no. 1, article 04016010, 2016.

[20] T. Lai, T.-H. Yi, and H.-N. Li, "Parametric study on sequential deconvolution for force identification," Journal of Sound and Vibration, vol. 377, pp. 76-89, 2016.

[21] T. Lai, T.-H. Yi, H.-N. Li, and X. Fu, "An explicit fourth-order Runge-Kutta method for dynamic force identification," International Journal of Structural Stability and Dynamics, vol. 17, no. 10, article 1750120, 2017.

[22] T. Lai, T.-H. Yi, and H.-N. Li, "Wavelet-Galerkin method for reconstruction of structural dynamic responses," Advances in Structural Engineering, vol. 20, no. 8, pp. 1174-1184, 2016.

[23] D. Bernal and A. Ussia, "Sequential deconvolution input reconstruction," Mechanical Systems and Signal Processing, vol. 50-51, pp. 41-55, 2015.

[24] M. Aucejo and O. De Smet, "On a full Bayesian inference for force reconstruction problems," Mechanical Systems and Signal Processing, vol. 104, pp. 36-59, 2018.

[25] B. Qiao, X. Zhang, J. Gao, and X. Chen, "Impact-force sparse reconstruction from highly incomplete and inaccurate measurements," Journal of Sound and Vibration, vol. 376, pp. 72-94, 2016. 
[26] Q. Li and Q. Lu, "A hierarchical Bayesian method for vibration-based time domain force reconstruction problems," Journal of Sound and Vibration, vol. 421, pp. 190-204, 2018.

[27] J. He, B. Xu, and S. F. Masri, "Restoring force and dynamic loadings identification for a nonlinear chain-like structure with partially unknown excitations," Nonlinear Dynamics, vol. 69 , no. 1-2, pp. 231-245, 2011.

[28] X. Xu and J. Ou, "An identification method of multi-source dynamic loads based on independent component analysis," Chinese Journal of Theoretical and Applied Mechanics, vol. 44, no. 1, pp. 158-166, 2012.

[29] J.-J. Liu, C.-K. Ma, I.-C. Kung, and D.-C. Lin, "Input force estimation of a cantilever plate by using a system identification technique," Computer Methods in Applied Mechanics and Engineering, vol. 190, no. 11-12, pp. 1309-1322, 2000.

[30] J.-S. Hwang, A. Kareem, and W.-J. Kim, "Estimation of modal loads using structural response," Journal of Sound and Vibration, vol. 326, no. 3-5, pp. 522-539, 2009.

[31] E. Lourens, E. Reynders, G. De Roeck, G. Degrande, and G. Lombaert, "An augmented Kalman filter for force identification in structural dynamics," Mechanical Systems and Signal Processing, vol. 27, pp. 446-460, 2012.

[32] F. Naets, J. Cuadrado, and W. Desmet, "Stable force identification in structural dynamics using Kalman filtering and dummy-measurements," Mechanical Systems and Signal Processing, vol. 50-51, pp. 235-248, 2015.

[33] E. N. Chatzi and C. Fuggini, "Structural identification of a super-tall tower by GPS and accelerometer data fusion using a multi-rate Kalman filter," in Proceedings of the 3th International Symposium on Life-Cycle Civil Engineering (IALCCE 2012), Vienna, Austria, October 2012.

[34] S. Eftekhar Azam, E. Chatzi, and C. Papadimitriou, "A dual Kalman filter approach for state estimation via output-only acceleration measurements," Mechanical Systems and Signal Processing, vol. 60-61, pp. 866-886, 2015.

[35] S. E. Azam, E. Chatzi, C. Papadimitriou, and A. Smyth, "Experimental validation of the Kalman-type filters for online and real-time state and input estimation," Journal of Vibration and Control, vol. 23, no. 15, pp. 2494-2519, 2015.

[36] S. Rapp, L. H. Kang, J. H. Han, U. C. Mueller, and H. Baier, "Displacement field estimation for a two-dimensional structure using fiber Bragg grating sensors," Smart Material Structures, vol. 18, no. 2, article 025006, 2009.

[37] E. Lourens, C. Papadimitriou, S. Gillijns, E. Reynders, G. De Roeck, and G. Lombaert, "Joint input-response estimation for structural systems based on reduced-order models and vibration data from a limited number of sensors," $\mathrm{Me}$ chanical Systems and Signal Processing, vol. 29, pp. 310-327, 2012.

[38] C. S. Hsieh, "Robust two-stage Kalman filters for systems with unknown inputs," IEEE Transactions on Automatic Control, vol. 45, no. 12, pp. 2374-2378, 2000. 




\section{Enfincering}
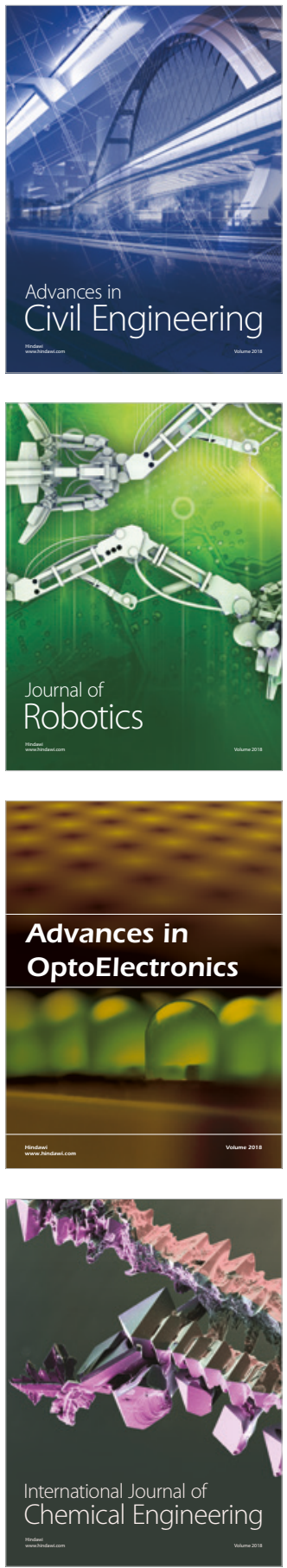



\section{Rotating \\ Machinery}

The Scientific World Journal

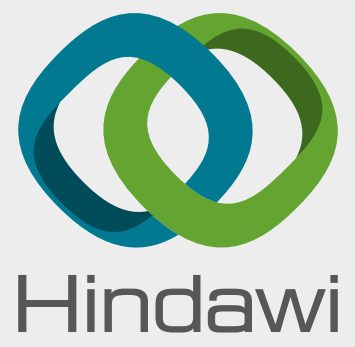

Submit your manuscripts at

www.hindawi.com
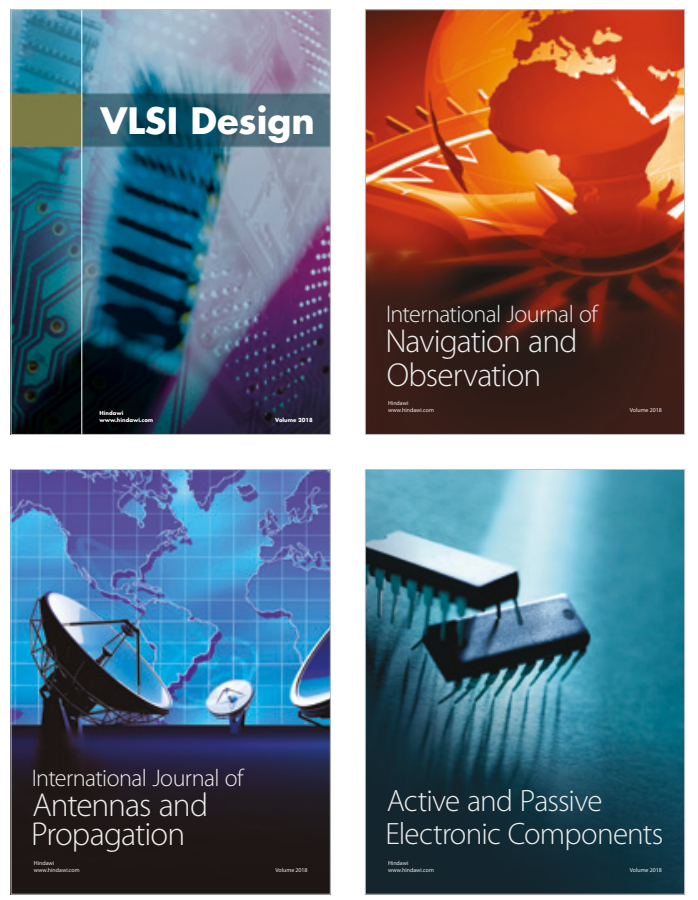
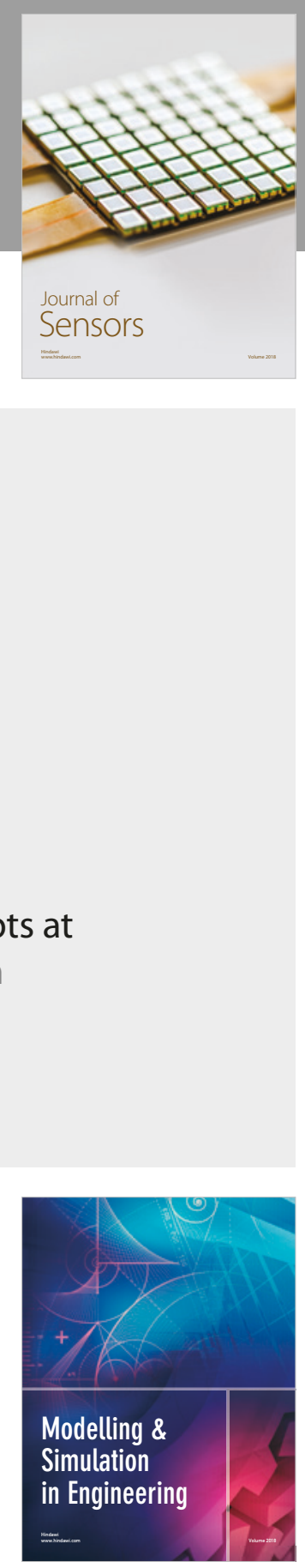

\section{Advances \\ Multimedia}
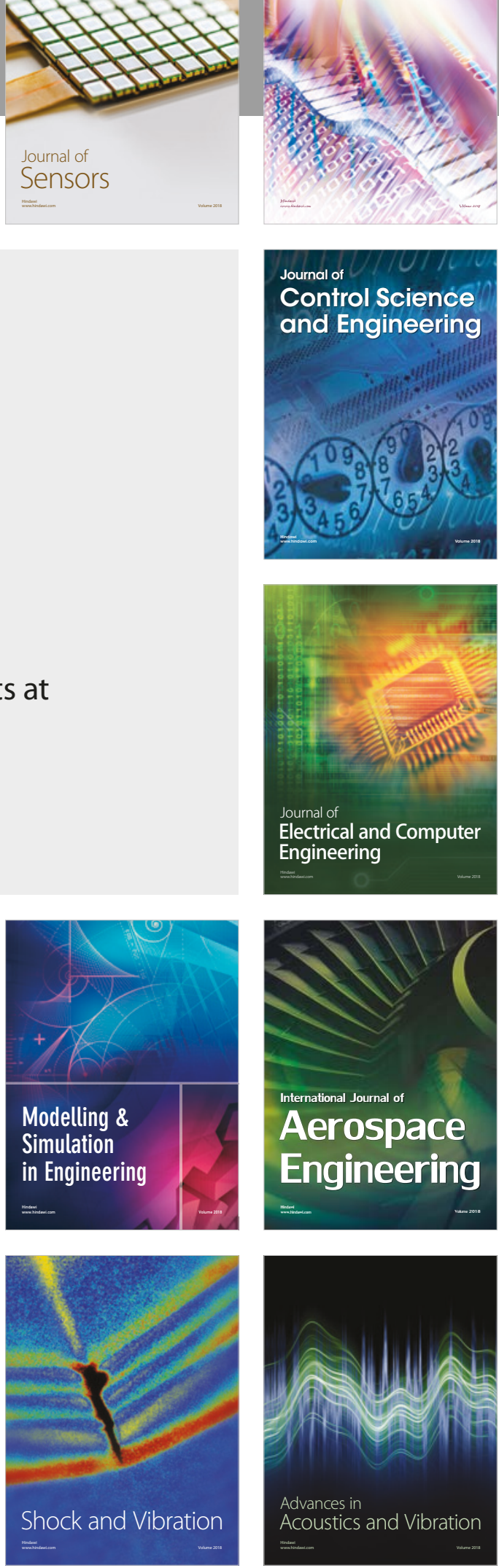